Maria de Lourdes Drachler 1,2

\title{
Desigualdade social e outros determinantes da altura em crianças: uma análise multinível
}

\author{
Social inequalities and other determinants \\ of height in children: a multi-level analysis
}

Márcia Christina Stark Andersson 3

José Carlos de Carvalho Leite 4

Tom Marshall 5

Denise Rangel Ganzo de Castro Aerts 6

Paulo Fontoura Freitas 7

Elsa Regina Justo Giuglianni 8

\footnotetext{
1 Programa de Pós-Graduação em Ciências da Saúde, Universidade do Vale do Rio dos Sinos. Av. Unisinos 950, São Leopoldo, RS 93022-000, Brasil. zecamalu@terra.com.br 2 Universidade Estadual do Rio Grande do Sul. Rua Gal. João Manoel 50, Porto Alegre, RS 90010-030, Brasil. 3 Departamento de Pediatria, Universi dade Católica de Pel otas. Rua Almirante Barroso 1202, Bloco G, Pelotas, RS 96010-280, Brasil.

4 Departamento de Psicologia, Universidade de Caxias do Sul. Rua Francisco Getúlio Vargas 1130, Caxias do Sul, RS 95070-560, Brasil.

5 London School of Hygiene and Tropical Medicine, London University. 50 Bedford Square, London WC1B3DP, UK.

6 Programa de Pós-Graduação em Saúde Coletiva,

Universidade Luterana do Brasil. Rua Miguel Tostes 101, Canoas, RS 92420-280, Brasil.

7 Programa de Mestrado em Ciências Médicas, Universidade Federal de Santa Catarina. Campus Universitário, Hospital Universitário, Florianópolis, SC 88040 970, Brasil.

8 Departamento de Pediatria, Universidade Federal do Rio Grande do Sul. Rua Ramiro Barcelos 2400 , Porto Alegre, RS

90035-003, Brasil.
}

Abstract A cross-sectional household survey of hei ght among children under fi ve years of age $(n=$ 2,632 ) was conducted in the city of Porto Al egre, Rio Grande do Sul State, Brazil. Multi-level linear regression was applied to investigate the effect of socioeconomic and demographic factors, physical and social environment, and health conditions on children's height, measured by the height-for-age z-scores of the National Center for Health Statistics standards. Area of resi dence (census tract) was classified as good versus poor in terms of housing and sanitation standards. On average, children's height was -0.18 z-score. Average height increased with maternal and paternal schooling, parents' work skills, per capita family income, improved housing, maternal age, birth intervals, and birth weight. Height decreased with hospitalization in thefirst two years of life, number of under-five children in the household, and preterm birth. In the poor residential areas, the effect of maternal schooling was twice as great as in the better-off areas. The effect of parental work skills was only evident in the more deprived areas. Area of residence modified the effects of soci oeconomic conditions on children's growth. Housing and sanitation programs are potentially beneficial to offset the negative effect of social disadvantage on children's growth. Key words Social Conditions; Child Development; Child Welfare

Resumo Estudo transversal de base populacional sobre altura de crianças de 12 a 59 meses ( $\mathrm{n}=$ 2.632) foi realizado em Porto Alegre, Brasil. Usou-se regressão linear multinível para investigar o efei to de condi ções sócio-econômi cas, demográficas, de saúde e dos ambientes físi co e social sobre a altura, medida em escores-z do padrão de altura para idade do National Center for Heal th Statisrics. A área de localização do domicílio foi classificada como bem e mal provida em infraestrutura habitacional. A al tura foi, em média, -0,18 escore-z, estando positivamente associada a escolaridade e qualificação ocupacional dos pais, renda, qualidade de moradia, idade materna, intervalo interpartal e peso de nascimento, e negativamente rel acionada a prematuridade, número de menores de cinco anos no domi cíli o e hospitalização nos dois primei ros anos de vida. O efeito da educação materna foi o dobro nas áreas mal providas em infra-estrutura habitacional. O efeito positivo da qualificação ocupacional dos pais foi evidente apenas nas áreas mal providas. Provavel mente, a área de residência modifica o efeito das condições sóci o-econômicas sobre o crescimento. Programas habitacionais e de saneamento são potencial mente úteis para diminuir o efei to de condições sócio-econômi cas desfavoráveis sobre o crescimento da criança. Palavras-chave Condições Sociais; Desenvolvimento Infantil; Bem-Estar da Criança 


\section{Intro dução}

A avaliação do crescimento da criança, com base em indicadores antropométricos, auxilia a análise do estado nutricional e de saúde individual e populacional (WHO, 1995). Relacionados ao retardo no crescimento encontramse: menor rendimento escolar (Lei et al., 1995; Malta et al., 1998), atraso no desenvolvimento (Drachler, 1998; Gopalan, 1987), imunocompetência reduzida (Scrimshaw \& SanGiovanni, 1997), maior risco de doença cardiovascular (Parker et al., 1998) e menor capacidade para o trabalho (Haas et al., 1996; Spurr, 1987). Mulheres com baixa estatura têm maior risco de terem filhos com baixo peso ao nascimento, fator de risco para retardo no crescimento infantil (Hernández-Díaz et al., 1999).

A tendência secular do crescimento e a prevalência de menor altura nos grupos desfavorecidos sócio-economicamente (Burrows et al., 1999; Kak, 1999; Prebeg, 1998) sugerem que o retardo no crescimento esteja mais relacionado à desnutrição de longa duração por privação alimentar ou morbidade reincidente (Waterlow, 1994) do que a fatores genéticos. Dentre os fatores sócio-econômicos, a escolaridade dos pais têm sido positivamente relacionada à altura da criança (Crooks, 1999; Drachler et al., 2002; Frongillo et al., 1997; Guimarães et al., 1999; Engstrom \& Anjos, 1999; Victora et al., 1986; Victora et al., 1992), provavelmente pelos melhores cuidados dedicados a ela e pelas maiores oportunidades profissionais, renda e acesso a bens e serviços (Crooks, 1999; Kronmeyer et al., 1997). Ocupação paterna manual (Goldstein, 1971; Prebeg, 1998; Smith et al., 1980), baixa renda familiar (Guimarães et al., 1999) e moradias inadequadas (Immink \& Payongayoung, 1999) têm sido associadas ao retardo no crescimento infantil. A baixa renda pode prejudicar o crescimento ao afetar a quantidade e a qualidade dos alimentos, o acesso aos serviços de saúde e a qualidade da moradia (Monteiro, 1988); já as moradias inadequadas afetam o crescimento porque aumentam o risco de infecções respiratórias, diarréia e outras doenças (D'Souza, 1997; Esrey et al, 1985; Gross et al., 1989; Immink \& Payongayong, 1999; Victora et al., 1986, 1988). Também associados ao retardo no crescimento infantil estão outros fatores mais freqüentes em famílias de baixa condição sócio-econômica, como intervalo interpartal curto e mais de uma criança pequena no domicílio (Kromeyer et al., 1997; Nóbrega et al.,1991; Victora et al., 1986), partos múltiplos maternos (Drachler et al., 2002), baixa idade materna (Berkowitz \& Papiernik, 1993;
Drachler et al., 2002), baixo peso de nascimento (Binkin et al., 1988; Drachler et al., 2002; Ricci \& Becker, 1996), prematuridade (Berkowitz \& Papiernik, 1993) e hospitalização da criança (Monteiro \& Benício, 1987). Os estudos revisados sugerem que as condições sócio-econômicas das famílias agem sobre o crescimento infantil, pelo menos em parte, porque influenciam o ambiente físico e social imediato e as condições de saúde da criança (Figura 1).

Este estudo investiga determinantes da altura em crianças e examina se o efeito das condições sócio-econômicas depen de da infra-estrutura habitacional da área de residência. $\mathrm{Na}$ revisão bibliográfica, somente um estudo (Desai \& Alva, 1998) investigou se o efeito de variáveis individuais sobre a saúde da criança depende de variáveis contextuais. Tal estudo, realizado em 22 países em desenvolvimento, mostrou que a educação materna tem maior efeito sobre a mortalidade infantil, a altura e o estado de imunização nas áreas geográficas onde menos de $75 \%$ das mães amostradas tinham educação secundária, sugerindo que as condições sócio-econômicas das famílias têm maior efeito sobre a saúde nas áreas menos favorecidas.

\section{Metodologia}

Este estudo utilizou dados de 2.632 crianças de 1 a 5 anos, do último inquérito sobre o estado nutricional e o desenvolvimento das crianças de Porto Alegre, realizado de agosto de 1988 a junho de 1990. A amostragem deu-se em três etapas: (a) amostragem sistemática de um sexto dos 1.646 setores censitários do município; (b) amostragem sistemática de domicílios de crianças menores de cinco anos em cada setor, selecionando um número de domicílios proporcional ao número de menores de cinco anos no setor e usando informações do Instituto Brasileiro de Geografia e Estatística (IBGE, 1983); (c) sorteio de uma criança em cada domicílio selecionado. Os pais deram consentimento informado. Das crianças de 12 a 59 meses selecionadas, 2,6\% não participaram do estudo por recusa ou não-localização da família e 23 foram excluídas: 14 por falta da medida da altura ou provável erro nessa medida, seis por paralisia cerebral grave, duas por síndrome de Down e uma por nanismo hipofisário.

A coleta de dados incluiu a medida do comprimento em menores de dois anos, realizada com antropômetro de madeira, e a da altura em maiores de dois anos, com fitas métricas de polietileno. As medidas seguiram procedimento padrão (Jelliffe, 1968; WHO, 1995). O escore 
de altura ou comprimento para idade e gênero foi calculado em escores- $z$ do padrão americano National Center for Health Statistics (NCHS, 1977), usando o módulo antropométrico EpiInfo versão 6,0 . Um questionário aplicado à mãe investigou escolaridade dos pais, ocupação da pessoa (pai ou mãe) com a maior renda no último ano, renda familiar per capita no último mês e história de hospitalização nos dois primeiros anos de vida. O peso ao nascimento foi copiado do cartão da criança recebido pela mãe na maternidade e, na falta deste, informado pela mãe. A idade gestacional foi coletada preferencialmente do cartão do recém-nascido ou calculada a partir da data provável do parto. Para crianças que viviam com a mãe biológica, foi investigada a idade da mãe ao nascimento da criança e o espaçamento interpartal em relação ao irmão anterior e ao posterior. As condições do domicílio foram codificadas em três grupos: os bem providos tinham eletricidade, saneamento básico (água potável e esgoto ligado à rede pública ou fossa) e acabamentos (assoalho e forro, portas e janelas com vidros e maçanetas); os inadequados tinham eletricidade e saneamento básico, mas faltava-Ihes algum acabamento; e os extremamente inadequados não tinham eletricidade ou saneamento básico. Os dois primeiros foram divididos em domicílios de tijolos, madeira ou mistos. A área de localização do domicílio (setor censitário) foi classificada como relativamente bem provida e mal provida em infra-estrutura habitacional. A mal provida tinha $30 \%$ ou mais de domicílios inadequados ou extremamente inadequados. A variável contextual infra-estrutura habitacional da área de domicílio foi gerada usando dados amostrais porque os setores censitários de 1991 não correspondiam aos de 1980 (cujos mapas foram usados para a coleta de dados deste estudo) e não foi possível compatibilizar as áreas dos setores dos dois censos, o que impossibilitou a utilização de variáveis investigadas no censo de 1991.

Todas as análises estatísticas (estimativa de médias e regressão linear) foram realizadas com técnica multinível, empregando-se o pacote estatístico MLn versão 10a (Goldstein, 1995). A análise multinível permite levar em conta (a) a estrutura hierárquica dos dados (crianças e setores) e (b) o interesse de estudar conjuntamente variáveis individuais (nível 1) e variáveis contextuais (infra-estrutura habitacional dos setores censitários, nível 2). O pacote estatístico MLn reconhece a estrutura hierárquica resultante da amostragem em dois níveis e, nesse caso, não assume que as observações sejam independentes. Análises que con-
Figura 1

Modelo hipotético dos efeitos das condições sócio-econômicas e outros fatores sobre a altura das crianças.

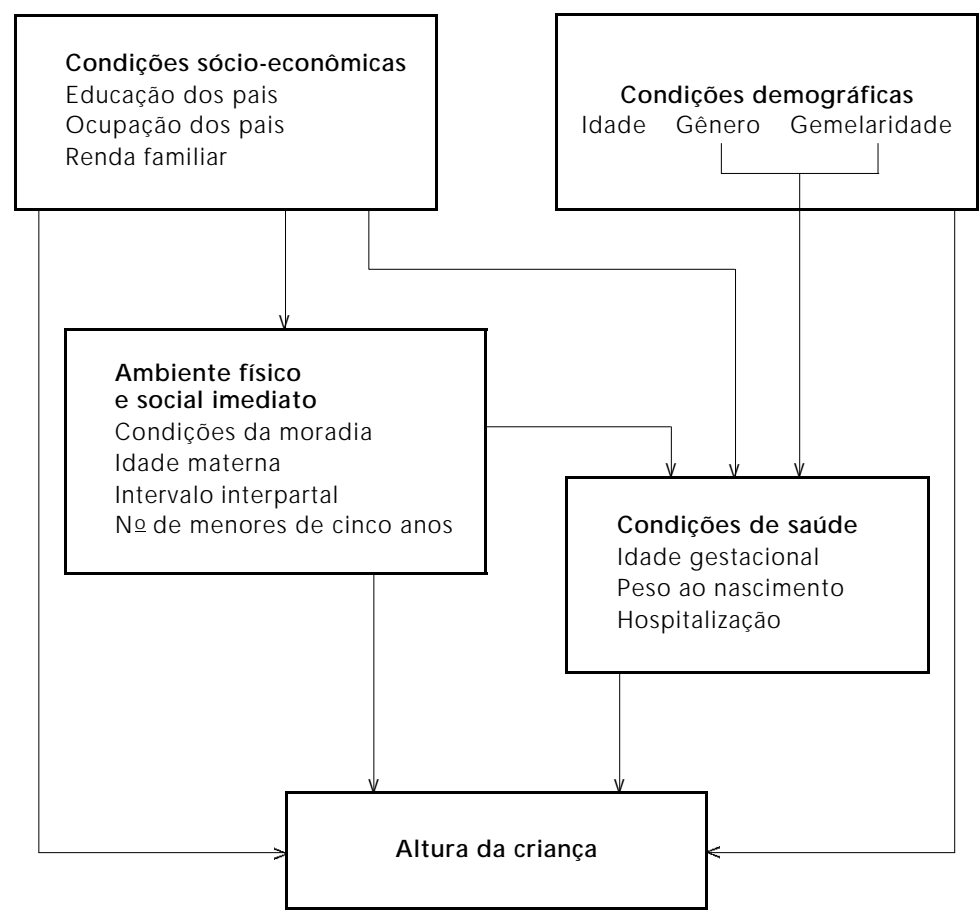

sideram os conglomerados, a exemplo do comando svyreg, do pacote estatístico Stata, também satisfazem o item (a), mas não o item (b), que requer modelagem multinível. A modelagem de nível 2 ajusta as estimativas da variação entre crianças para a variação entre os setores censitários e dentro destes, dando mais peso às comparações no mesmo setor, já que as crianças da mesma área tendem a ser mais parecidas entre si do que as moradoras em áreas diferentes

Regressão linear multinível com as variáveis de exposição modeladas como variáveis dummy foi usada para estimar diferenças em altura entre as categorias das variáveis de exposição, em relação à categoria de menor risco esperado. A exceção foi o peso ao nascimento, cuja referência foi a categoria de peso mediano da amostra. Foram calculados coeficientes de regressão brutos (não apresentados) e ajustados para confundimento. A escolha dos possíveis confundidores de cada variável baseou-se no modelo teórico apresentado na Figura 1. Nessa figura, as variáveis distribuem-se 
em níveis de determinação da altura, de modo que os possíveis mediadores do efeito de cada variável localizem-se abaixo dela, e os possíveis confundidores do seu efeito, acima ou ao lado dela. Por exemplo, o número de menores de cinco anos no domicílio é um possível mediador do intervalo interpartal, pois parte do efeito do espaçamento interpartal curto pode dever-se ao maior número de crianças pequenas no domicílio. Assim, conforme Weinberg (1993), o intervalo interpartal não pode ser ajustado pelo número de menores de cinco anos. A exceção ocorreu para condições do domicílio e idade materna que, embora no mesmo nível do intervalo interpartal, foram ajustadas pelo número de menores de cinco anos porque não se espera que o efeito negativo da baixa idade materna e das moradias inadequadas deva-se ao número de crianças pequenas.

Foram investigadas somente as interações hipotéticas a priori, ou seja, as interações entre a infra-estrutura habitacional da área de residência e as condições sócio-econômicas da criança. As interações foram examinadas de duas maneiras: primeiro modelando a exposição sócio-econômica de interesse como variável dummy e depois como tendência linear do escore de altura-para-idade através dos grupamentos da variável sócio-econômica codificados como escores ordenados, por exemplo, 1,2,3,4 para uma série de 4 categorias. (Os dois modelos podem ser utilizados porque essas variáveis são intervalares ou suas categorias estão em uma ordem natural.) As demais variáveis de cada modelo foram modeladas como variáveis dummy. O método da razão de verossimilhança (Greenland \& Rothman, 1998) foi usado para calcular a significância estatística da diferença na bondade do ajuste entre os dois modelos. Quando a diferença na razão de verossimilhança entre os modelos não foi estatisticamente significante ( $p>0,05)$, não houve evidência de que o modelo com variáveis dummy se ajustasse melhor aos dados do que o modelo linear. Nessa situação, foi apresentada a significância estatística do modelo de tendência linear porque dá maior poder para o estudo evidenciar a interação. Quando o teste da razão de verossimilhança foi estatisticamente significante $(p<0,05)$, foi apresentada a significância estatística do modelo com variáveis dummy.

As significâncias estatísticas das variáveis de exposição e das interações modeladas como variáveis dummy foram calculadas pelo método razão de verossimilhança, comparando-se o modelo que inclui e o que exclui a variável ou interação de interesse (Greenland \& Rothman,
1998). As significâncias das exposições modeladas como variáveis dicotômicas e das interações modeladas como tendência linear foram calculadas pelo teste-t. Preestabeleceu-se o nível de significância estatística de 5\%, exceto para as interações, preestabelecidas em $10 \%$, em virtude do baixo poder dos testes para evidenciá-las.

Os modelos de regressão mostraram-se estatisticamente adequados, como indicado pela normalidade e ausência de observações extremas nos histogramas dos resíduos estandardizados (níveis 1 e 2), assim como pela ausência de associação entre tais resíduos e os valores preditos pela regressão investigada em diagramas de dispersão. A análise dos resíduos foi realizada para o modelo multivariado mais simples, que incluiu escolaridade materna e paterna e variáveis demográficas, e para o modelo com todas as variáveis de exposição estudadas.

\section{Resultados}

A desigualdade social foi grande. Cerca de $40 \%$ das famílias moravam em áreas mal providas em infra-estrutura habitacional. Nessas áreas, $44 \%$ das mães tinham menos de cinco anos de escolaridade, enquanto nas áreas bem providas essa proporção foi de $10 \%$ (Tabela 1). Um trabalhador não qualificado era o principal responsável pela renda em $50 \%$ das famílias nas áreas mal providas, e em $20 \%$ nas bem providas. A renda era inferior a 0,5 salário mínimo per capita em $18 \%$ das famílias, e em $30 \%$ as moradias eram inadequadas ou extremamente inadequadas (Tabelas 2 e 3 ).

O índice de altura para idade foi, em média, -0,18 escores-z do padrão NCHS (não apresentado em tabela). A média de altura aumentou com a escolaridade e a qualificação profissional dos pais, a renda familiar per capita, a qualidade do domicílio, a idade materna ao nascimento da criança, o intervalo interpartal e o peso ao nascimento. As diferenças em altura associadas à escolaridade materna e à ocupação dos pais foram maiores nas áreas mal providas em infra-estrutura habitacional. A média de altura foi menor à medida que era maior o número de menores de cinco anos no domicílio e o tempo de hospitalização nos dois primeiros anos de vida; foi menor também entre as crianças que haviam nascido prematuras ou de gestação múltipla (Tabelas 1 a 4).

Os modelos de regressão ajustados para confundimento mostram que o efeito positivo da escolaridade materna diferiu entre as áreas de mo- 
Número de crianças, médias de altura para idade e coeficientes de regressão linear das diferenças entre essas médias para educação paterna e para educação materna em áreas bem e mal providas em infra-estrutura habitacional.

\begin{tabular}{|c|c|c|c|c|c|c|}
\hline & \multicolumn{2}{|c|}{ Crianças } & \multicolumn{2}{|c|}{ Altura para idade } & \multicolumn{2}{|c|}{ Regressão linear } \\
\hline & $\mathrm{n}$ & $\%$ & Média & IC $95 \%$ & Coeficientes & $5 \quad I C 95 \%$ \\
\hline \multicolumn{7}{|c|}{ Educação paterna1 (anos) } \\
\hline$\geq 12$ & 501 & 19,0 & 0,32 & 0,23 a 0,41 & & 0 \\
\hline $9-11$ & 544 & 20,7 & 0,07 & $-0,03$ a $-0,16$ & $-0,10$ & $-0,23$ a 0,04 \\
\hline $5-8$ & 774 & 28,3 & $-0,30$ & $-0,43$ a $-0,16$ & $-0,22$ & $-0,36$ a $-0,07$ \\
\hline $1-4$ & 348 & 13,2 & $-0,69$ & $-0,83$ a $-0,55$ & $-0,37$ & $-0,55$ a $-0,19$ \\
\hline$<1$ & 97 & 3,7 & $-0,84$ & $-1,06$ a $-0,61$ & $-0,39$ & $-0,65$ a $-0,12$ \\
\hline Ausência so pai & 368 & 14,0 & $-0,35$ & $-0,47$ a $-0,23$ & $-0,30$ & $-0,46$ a $-0,14$ \\
\hline \multirow[t]{2}{*}{ Total } & 2.632 & & & & & \\
\hline & & & & & & $p<0,001$ \\
\hline \multicolumn{7}{|c|}{ Educação materna1 (anos) } \\
\hline \multicolumn{7}{|c|}{ Áreas bem providas } \\
\hline$\geq 12$ & 443 & 27,8 & 0,36 & 0,27 a 0,45 & & 0 \\
\hline $9-11$ & 491 & 31,0 & 0,10 & 0,01 a 0,19 & $-0,19$ & $-0,33$ a $-0,04$ \\
\hline $5-8$ & 496 & 30,8 & 0,02 & $-0,08$ a 0,11 & $-0,18$ & $-0,34$ a $-0,02$ \\
\hline $1-4$ & 137 & 8,6 & $-0,33$ & $-0,54$ a $-0,12$ & $-0,46$ & $-0,69$ a $-0,23$ \\
\hline$<1$ & 25 & 1,6 & $-0,11$ & $-0,58$ a $-0,35$ & $-0,33$ & $-0,77$ a 0,11 \\
\hline Total & 1.592 & & & & & \\
\hline \multicolumn{7}{|l|}{ Áreas mal providas } \\
\hline$\geq 12$ & 42 & 4,0 & 0,09 & $-0,20$ a 0,39 & & 0 \\
\hline $9-11$ & 118 & 11,3 & $-0,28$ & $-0,48$ a $-0,09$ & $-0,20$ & $-0,58$ a 0,18 \\
\hline $5-8$ & 425 & 40,9 & $-0,53$ & $-0,62$ a $-0,43$ & $-0,36$ & $-0,71$ a $-0,01$ \\
\hline $1-4$ & 344 & 33,0 & $-0,79$ & $-0,91$ a $-0,66$ & $-0,55$ & $-0,91$ a $-0,19$ \\
\hline$<1$ & 111 & 10,7 & $-1,12$ & $-1,35$ a $-0,90$ & $-0,84$ & $-1,23$ a $-0,44$ \\
\hline Total & 1.040 & & & & & \\
\hline $\begin{array}{l}\text { Interação como } \\
\text { tendência linear }\end{array}$ & & & & & & $p=0,052$ \\
\hline
\end{tabular}

1 Ajustado para idade, sexo, gemelaridade e outra variável na tabela.

radia (interação sumarizada como tendência linear, $p=0,052$ ). Nas áreas mal providas, a escolaridade materna teve o dobro do efeito estimado para as áreas bem providas, prevendo-se que os filhos de mães analfabetas tenham um déficit de altura de 0,8 escore- $z$, comparados ao grupo com a mais alta escolaridade materna (Tabela 1). A ocupação dos pais foi outra variável cujo efeito diferiu entre áreas (interação como tendência linear; $p=0,038$ ). Somente nas áreas mal providas houve evidência de que a altura da criança era maior à medida que a ocupação dos pais era mais qualificada. Os modelos multivariados mostram que a escolaridade paterna e a renda familiar per capita tiveram efeito positivo sobre a altura das crianças (Tabelas 1 e 2), mas não houve evidência de interação entre essas variáveis e a infra-estrutura habitacional da área (escolaridade paterna, interação como tendência linear $p=0,290$; ren- da, interação como variáveis dummy $p=0,670$; não apresentados em tabela).

Os coeficientes de regressão ajustados mostram ainda que a altura foi maior à medida meIhoravam as condições de moradia da criança e foi menor para os gêmeos, os filhos de mãe adolescente, as crianças cujo intervalo interpartal em relação ao irmão anterior e ao posterior era menor de 24 meses e quando moravam mais de dois menores de cinco anos no domicílio. Comparado ao padrão NCHS, houve déficit de altura para ambos os sexos, sendo maior o déficit entre os meninos (Tabela 3 ). Também relevantes foram os efeitos da prematuridade, hospitalização e peso ao nascimento. Esta foi a variável de maior efeito sobre a altura, estimando-se que os nascidos com menos de $2.000 \mathrm{~g}$ eram, em média, 1,2 escores- $z$ mais baixos do que os nascidos com mais de $4.000 \mathrm{~g}$ (Tabela 4) 
Número de crianças, médias de altura para idade e coeficientes de regressão linear das diferenças entre essas médias para qualificação profissional dos pais em áreas bem e mal providas em infra-estrutura habitacional e para renda familiar per capita.

\begin{tabular}{|c|c|c|c|c|c|c|}
\hline & \multicolumn{2}{|c|}{ Crianças } & \multicolumn{2}{|c|}{ Altura para idade } & \multicolumn{2}{|c|}{ Regressão linear } \\
\hline & $\mathrm{n}$ & $\%$ & Média & IC $95 \%$ & Coeficientes & $5 \quad I C 95 \%$ \\
\hline \multicolumn{7}{|l|}{ Qualificação ocupacional1 } \\
\hline \multicolumn{7}{|l|}{ Áreas bem providas } \\
\hline Empregadores/graduados & 501 & 31,4 & 0,28 & 0,19 a 0,36 & & 0 \\
\hline Outros qualificados & 743 & 46,7 & 0,09 & 0,01 a 0,17 & $-0,01$ & $-0,15$ a 0,13 \\
\hline Manual não qualificado & 300 & 18,8 & $-0,08$ & $-0,21$ a 0,05 & $-0,03$ & $-0,21$ a 0,15 \\
\hline Sem trabalho $>3$ meses & 48 & 3,0 & $-0,32$ & $-0,59$ a $-0,04$ & $-0,29$ & $-0,63$ a 0,05 \\
\hline Total & 1.592 & & & & & \\
\hline \multicolumn{7}{|l|}{ Áreas mal providas } \\
\hline Empregadores/graduados & 68 & 6,5 & $-0,19$ & $-0,42$ a 0,05 & & 0 \\
\hline Outros qualificados & 436 & 42,0 & $-0,45$ & $-0,55$ a $-0,34$ & $-0,04$ & $-0,32$ a 0,23 \\
\hline Manual não qualificado & 499 & 48,0 & $-0,83$ & $-0,93$ a $-0,73$ & $-0,25$ & $-0,53$ a 0,04 \\
\hline Sem trabalho $>3$ meses & 37 & 3,5 & $-0,72$ & $-1,04$ a $-0,39$ & $-0,16$ & $-0,60$ a 0,28 \\
\hline Total & 1.040 & & & & & \\
\hline $\begin{array}{l}\text { Interação como tendência } \\
\text { linear (excluindo os sem } \\
\text { trabalho) }\end{array}$ & & & & & & $p=0,038$ \\
\hline \multicolumn{7}{|l|}{ Renda per capita 2} \\
\hline$\geq 2,0$ salários & 915 & 35,0 & 0,26 & 0,19 a 0,32 & & 0 \\
\hline 1,0-1,9 salários & 580 & 22,0 & $-0,03$ & $-0,12$ a 0,12 & $-0,12$ & $-0,25$ a 0,01 \\
\hline $0,5-0,9$ salários & 653 & 24.8 & $-0,41$ & $-0,50$ a $-0,33$ & $-0,32$ & $-0,46$ a $-0,18$ \\
\hline$<0,5$ salário & 477 & 18,0 & $-0,90$ & $-1,00$ a $-0,39$ & $-0,63$ & $-0,80$ a $-0,47$ \\
\hline \multirow[t]{2}{*}{ Total } & 2.625 & & & & & \\
\hline & & & & & & $p<0,001$ \\
\hline
\end{tabular}

1 Ajustado para idade, gênero, gemelaridade, educação paterna e educação materna por áreas bem e mal providas em infra-estrutura habitacional.

2 Ajustado para idade, gênero, gemelaridade, educação paterna e educação materna por áreas bem e mal providas em infra-estrutura habitacional e qualificação profissional por áreas.

\section{Discussão}

Os resultados desta pesquisa alinham-se aos de estudos que mostram a importância da determinação social do crescimento infantil, particularmente a da escolaridade materna (Bobak et al., 1994; Carvalhaes \& Benício, 2002, Drachler et al., 2002; Engstrom \& Anjos, 1999; Frongillo et al., 1997; Guimarães et al., 1999; Victora et al., 1992), escolaridade paterna (Crooks, 1999; Drachler et al., 2002; Victora et al., 1986), qualificação ocupacional dos pais (Goldstein, 1971; Prebeg, 1998) e renda familiar (Guimarães et al., 1999). O efeito da escolaridade materna e da ocupação dos pais dependeu da infra-estrutura habitacional da área de moradia. A escolaridade teve maior efeito nas áreas mal providas, resultado semelhante ao de um estudo realizado em várias áreas de 22 paí- ses em desenvolvimento (Desai \& Alva, 1998). Também em outro estudo o efeito da escolaridade materna sobre a altura de crianças foi maior em Porto Alegre do que nas cinco maiores cidades da república Checa, onde todas as famílias moravam em domicílios com saneamento básico e as desigualdades sócio-econômicas eram bem menores do que na amostra brasileira (Drachler et al., 2002). É possível que o efeito da escolaridade materna ocorra por vários caminhos, desde melhores condições de saúde ao nascimento, práticas nutricionais e de higiene adequadas, além de outras que promovam e preservem a saúde infantil (Cleland \& van Ginneken, 1988; Guldan et al., 1993; Muñiz \& Rubi, 1988). A ocupação dos pais, indicador da posição social e um determinante da renda familiar, associou-se à altura da criança somente nas áreas mal providas, provavelmen- 
Crianças, médias de altura para idade e coeficientes de regressão linear das diferenças entre as médias para ambiente físico e social imediato e variáveis demográficas.

\begin{tabular}{|c|c|c|c|c|c|c|}
\hline & \multicolumn{2}{|c|}{ Crianças } & \multicolumn{2}{|c|}{ Altura para idade } & \multicolumn{2}{|c|}{ Regressão linear } \\
\hline & $\mathrm{n}$ & $\%$ & média & IC $95 \%$ & coeficientes & S IC $\quad$ IC $5 \%$ \\
\hline \multicolumn{7}{|l|}{ Condições do domicílio 1} \\
\hline Adequado, tijolos & 1.497 & 59,0 & 0,12 & 0,07 a 0,17 & 0 & \\
\hline Adequado, madeira e misto & 319 & 12,6 & $-0,06$ & $-0,18$ a 0,06 & $-0,01$ & $-0,14$ a 0,12 \\
\hline Inadequado, tijolos & 153 & 6,0 & $-0,42$ & $-0,57$ a $-0,27$ & $-0,10$ & $-0,29$ a 0,09 \\
\hline Inadequado, madeira ou misto & 236 & 9,4 & $-0,79$ & $-0,94$ a $-0,65$ & $-0,36$ & $-0,53$ a $-0,19$ \\
\hline Extremamente inadequado & 316 & 12,5 & $-1,06$ & $-1,19$ a $-0,93$ & $-0,50$ & $-0,67$ a $-0,32$ \\
\hline \multirow[t]{2}{*}{ Total } & 2.521 & & & & & \\
\hline & & & & & & $p<0,001$ \\
\hline \multicolumn{7}{|l|}{ Idade materna (anos) 1} \\
\hline $12-19$ & 310 & 12,6 & $-0,58$ & $-0,70$ a $-0,45$ & $-0,18$ & $-0,31$ a $-0,05$ \\
\hline $20-34$ & 1.841 & 75,0 & $-0,11$ & $-0,16$ a $-0,06$ & 0 & \\
\hline $35-50$ & 301 & 12,3 & $-0,06$ & $-0,21$ a 0,08 & 0,08 & $-0,05$ a 0,21 \\
\hline \multirow[t]{2}{*}{ Total } & 2.452 & & & & & \\
\hline & & & & & & $p=0,008$ \\
\hline \multicolumn{7}{|l|}{ Intervalo interpartal2 } \\
\hline$<24$ meses & 398 & 16,2 & $-0,57$ & $-0,69$ a 0,44 & $-0,30$ & $-0,44$ a $-0,16$ \\
\hline 24-47 meses & 578 & 23,6 & $-0,27$ & $-0,37$ a $-0,18$ & $-0,19$ & $-0,31$ a $-0,07$ \\
\hline$\geq 48$ meses & 641 & 26,0 & $-0,04$ & $-0,13$ a 0,05 & 0 & \\
\hline Filho único & 835 & 34,0 & 0,01 & $-0,07$ a 0,08 & 0,05 & $-0,16$ a 0,07 \\
\hline \multirow[t]{2}{*}{ Total } & 2.452 & & & & & \\
\hline & & & & & & $p<0,001$ \\
\hline \multicolumn{7}{|l|}{ Menores de 5 anos 1} \\
\hline 1 & 1.964 & 78,0 & $-0,08$ & $-0,13$ a $-0,03$ & 0 & \\
\hline 2 & 481 & 19,0 & $-0,39$ & $-0,50$ a $-0,29$ & 0,07 & $-0,05$ a 0,19 \\
\hline$\geq 3$ & 76 & 3,0 & $-1,06$ & $-1,34$ a $-0,78$ & $-0,30$ & $-0,54$ a $-0,06$ \\
\hline \multirow[t]{2}{*}{ Total } & 2.521 & & & & & \\
\hline & & & & & & $p=0,010$ \\
\hline \multicolumn{7}{|l|}{ Gênero 1} \\
\hline Masculino & 1.284 & 51,0 & $-0,21$ & $-0,27$ a $-0,14$ & $-0,082$ & $-0,16$ a $-0,01$ \\
\hline Feminino & 1.237 & 49,0 & $-0,13$ & $-0,19$ a $-0,06$ & 0 & \\
\hline \multirow[t]{2}{*}{ Total } & 2.521 & & & & & \\
\hline & & & & & & $p=0,045$ \\
\hline \multicolumn{7}{|l|}{ Gemelaridade 1} \\
\hline Não & 2.489 & 99,0 & $-0,16$ & $-0,21$ a $-0,18$ & 0 & \\
\hline Sim & 32 & 1,0 & $-0,66$ & $-1,08$ a $-0,25$ & $-0,48$ & $-0,84$ a $-0,11$ \\
\hline \multirow[t]{2}{*}{ Total } & 2.521 & & & & & \\
\hline & & & & & & $p=0,010$ \\
\hline
\end{tabular}

1 Modelo inclui idade, sexo, gemelaridade, educação materna por áreas bem e mal providas em infra-estrutura habitacional, educação paterna, qualificação profissional por áreas, renda, moradia, idade materna, intervalo interpartal e menores de cinco anos

2 Modelo inclui idade, sexo, gemelaridade, educação materna por áreas bem e mal providas em infra-estrutura habitacional, educação paterna, qualificação profissional por áreas, renda, moradia, idade materna (excluindo menores de cinco anos, possível mediador do intervalo interpartal). 
N úmero de crianças, médias de altura para idade e coeficientes de regressão linear das diferenças entre essas médias para as condições de saúde da criança.

\begin{tabular}{|c|c|c|c|c|c|c|}
\hline & \multicolumn{2}{|c|}{ Crianças } & \multicolumn{2}{|c|}{ Altura para idade } & \multicolumn{2}{|c|}{ Regressão linear } \\
\hline & $\mathrm{n}$ & $\%$ & Média & IC $95 \%$ & Coeficien & e $\quad$ IC $95 \%$ \\
\hline Idade gestacional1 & & & & & & \\
\hline$\geq 37$ semanas & 2.401 & 95,0 & $-0,15$ & $-0,19$ a $-0,10$ & & 0 \\
\hline$<37$ semanas & 120 & 4,7 & $-0,62$ & $-0,81$ a $-0,43$ & $-0,49$ & $-0,68$ a $-0,30$ \\
\hline \multirow[t]{2}{*}{ Total } & 2.521 & & & & & \\
\hline & & & & & & $p<0,001$ \\
\hline \multicolumn{7}{|c|}{ Peso ao nascimento 2} \\
\hline$<2.000 \mathrm{~g}$ & 56 & 2,2 & $-1,01$ & $-1,34$ a $-0,68$ & $-0,64$ & $-0,93$ a $-0,34$ \\
\hline $2.000-2.499 \mathrm{~g}$ & 148 & 5,8 & $-0,64$ & $-0,83$ a $-0,44$ & $-0,36$ & $-0,54$ a $-0,18$ \\
\hline $2.500-2.999 \mathrm{~g}$ & 545 & 21,6 & $-0,51$ & $-0,61$ a $-0,42$ & $-0,28$ & $-0,39$ a $-0,18$ \\
\hline $3.000-3.499 \mathrm{~g}$ & 934 & 37,0 & $-0,14$ & $-0,21$ a $-0,07$ & & 0 \\
\hline $3.500-3.999 g$ & 634 & 25,0 & 0,07 & $-0,03$ a 0,16 & 0,22 & 0,12 a 0,32 \\
\hline$\geq 4.000 \mathrm{~g}$ & 204 & 8,0 & 0,46 & 0,32 a 0,60 & 0,59 & 0,44 a 0,74 \\
\hline \multirow[t]{2}{*}{ Total } & 2.521 & & & & & \\
\hline & & & & & & $p<0,001$ \\
\hline \multicolumn{7}{|c|}{ Hospitalização (semanas)³ } \\
\hline $0-0,9$ & 2.124 & 84,0 & $-0,07$ & $-0,11$ a $-0,02$ & & 0 \\
\hline $1-1,9$ & 176 & 7,0 & $-0,57$ & $-0,75$ a $-0,38$ & $-0,22$ & $(-0,37$ a $-0,06)$ \\
\hline $2-6$ & 180 & 7,0 & $-0,70$ & $-0,88$ a $-0,51$ & $-0,14$ & $-0,30$ a 0,02 \\
\hline$\geq 7$ & 41 & 1,6 & $-1,45$ & $-1,85$ a $-1,06$ & $-0,66$ & $-0,97$ a $-0,35$ \\
\hline \multirow[t]{2}{*}{ Total } & 2.521 & & & & & \\
\hline & & & & & & $p<0,001$ \\
\hline
\end{tabular}

1 Modelo de regressão inclui idade, sexo, gemelaridade, educação materna por áreas bem e mal providas em infra-estrutura habitacional, educação paterna, qualificação profissional por áreas, renda, moradia, idade materna, intervalo interpartal, menores de cinco anos e idade gestacional.

2 Modelo de regressão inclui idade, sexo, gemelaridade, educação materna por áreas bem e mal providas

em infra-estrutura habitacional, educação paterna, qualificação profissional por áreas, renda, moradia, idade materna, intervalo interpartal, menores de cinco anos, idade gestacional e peso de nascimento.

3 Modelo de regressão inclui idade, sexo, gemelaridade, educação materna por áreas bem e mal providas

em infra-estrutura habitacional, educação paterna, qualificação profissional por áreas, renda, moradia, idade materna, intervalo interpartal, menores de cinco anos e idade gestacional, peso de nascimento e hospitalização.

te porque a ocupação mais qualificada viabilize a aquisição de bens e serviços de melhor qualidade, usualmente não disponíveis aos moradores daquelas áreas.

Os resultados deste estudo indicam a importância dos ambientes físico e social no processo do crescimento. A altura tendia a ser menor em crianças que viviam em domicílios com infra-estrutura inadequada e em filhos de mães adolescentes e com alta fecundidade, indicada por intervalo interpartal curto e mais de duas crianças menores de cinco anos no domicílio. Domicílios inadequados aumentam o risco de doenças (Binkin et al., 1988; D'Souza, 1997; Esrey et al., 1985; Gross et al., 1989; Immink \& Payongayong, 1999; Victora et al., 1986, 1988), sendo a falta de água potável e de saneamento básico um dos maiores determinantes de retardo no crescimento de crianças (Immink \&
Payongayoung, 1999). A maternidade em idade precoce associa-se a inexperiência no manuseio da criança, menor percepção de suas necessidades e menor agilidade em situação de doença. O alto número de partos pode diminuir a disponibilidade materna e a quantidade intradomiciliar de alimentos (Nóbrega et al., 1991). O peso ao nascimento, maior determinante da altura neste estudo, tem demonstrado ser um dos maiores preditores da altura da criança, juntamente com a altura dos pais, que reflete fatores genéticos e ambientais (Bielicki, 1986), variável que não foi considerada no presente estudo devido à falta dessa medida. O efeito da prematuridade e o do peso ao nascimento, também evidentes em outros estudos (Binkin et al., 1988; Ricci \& Becker, 1996), podem dever-se em parte a dificuldades no estabelecimento da alimentação e complicações 
clínicas (Berkowitz \& Papiernik, 1993). O efeito negativo da hospitalização pode refletir doenças mais graves, principalmente diarréia e doenças respiratórias (Issler et al., 1996; Silva et al., 1999; Victora et al., 1992), mais freqüentes nos primeiros anos de vida (Monteiro, 1988), período em que a necessidade nutricional e a velocidade de crescimento são maiores (Martorell et al., 1994).

Este estudo avaliou apenas uma criança em cada domicílio selecionado porque na época da coleta de dados (1988-1990) não se dispunha de pacotes estatísticos com comandos que permitissem levar em conta os conglomerados, nem de pacotes estatísticos multiníveis. Sem esses recursos estatísticos, se todas as crianças de cada domicílio selecionado fossem incluídas na amostra, provavelmente haveria superestimação dos coefi cientes de regressão para as variáveis individuais, já que se previa a utilização de modelos de regressão tradicionais. Por outro lado, essa opção não permite investigar efeitos do "conglomerado família" sobre a altura das crianças. Outros estudos que investiguem todas as crianças de cada domicílio selecionado poderão fornecer contribuição adicional ao conhecimento do processo de crescimento.

\section{Agradecimentos}

Financiado pela Fundação de Amparo à Pesquisa do Estado do Rio Grande do Sul, Escola de Saúde Pública do Estado do Rio Grande do Sul, Secretarias Municipais de Saúde de Porto Alegre e Novo Hamburgo, Conselho Nacional de Desenvolvimento Científico e Tecnológico e Fundo de Incentivo à Pesquisa do Hospital de Clínicas de Porto Alegre.
Os resultados do presente estudo sugerem que investimentos governamentais em educação, qualificação profissional e outros que aumentem a renda familiar e a qualidade das moradias são potencialmente úteis para, a médio e longo prazos, melhorarem a saúde das crianças. Embora a avaliação das áreas de moradia tenha se baseado em medidas da própria amostra e a infra-estrutura habitacional adequada possa refletir a existência na área de outros fatores benéficos à saúde e ao crescimento infantil não controlados neste estudo (outros serviços públicos e privados na área), é provável que programas habitacionais para famílias de baixa renda e programas de saneamento básico possam, a curto prazo, diminuir o efeito da baixa escolaridade e da ocupação não qualificada dos pais sobre a saúde e o crescimento infantis. Os profissionais de saúde devem estar atentos para tratar precocemente desvios nutricionais e preveni- los mediante ações de planejamento familiar, atenção pré-natal e à saúde da mulher e da criança, especialmente relevantes para famílias em piores condições sócio-econômicas.

\section{Referências}

BERKOWITZ, G. S. \& PAPIERNIK, E., 1993. Epidemiology of preterm birth. Epidemiologic Reviews, 15:414-443.

BINKIN, N. J.; YIP, R.; FLESHHOOD, L. \& TROWBRIDGE, F. L., 1988. Birth weight and childhood growth. Pediatrics, 82:828-834.

BIELICKI, T., 1986. Physical growth as a measure of the economic well-being of populations: The twentieth century. In: Human Growth. A Comprehensi ve Treatise Methodology, Ecological, Genetic, and Nutritional Effects on Growth (F. Falkner \& J. M. Tanner, ed.), pp. 283-305, v. 3, 2nd Ed. New York: Plenum Press,

BOBAK, M.; KRIZ, B.; LEON, D. A.; DANOVA, J. \& MARM OT, M., 1994. Socioeconomic factors and height of preschool children in the Czech Republic. American Journal of Public Health, 84:11671170.

BURROWS, R.; RAMIREZ, I.; CORDERO, J. \& MUZZO, S., 1999. Cambio secular del retraso de talla em escolares chilenos de tres regiones del país. $\mathrm{Re}$ vista Chilena de Pediatría, 70:390-397. 
CARVAlHAES, M. A. \& BeNíCIO, M. H., 2002. Capacidade materna de cuidar e desnutrição infantil. Revista de Saúde Pública, 36:188-197.

CLELAND, J. G. \& van GINNEKEN, J. K., 1988. Maternal education and child survival in developing countries: The search for pathways of influence. Social Science and Medicine, 27:1357-1368.

CROOKS, D. L., 1999. Child growth and nutritional status in a high-poverty community in eastern Kentucky. American Journal of Physical Anthropology, 109:129-142.

DESAI, S. \& ALVA, S., 1998. Maternal education and child health: Is there a strong causal relationship? Demography, 35:71-81.

DRACHLER, M. L., 1998. Social Inequalities in Child Devel opment: A Cross-sectional Survey on Developmental Determinants Among Children Aged 6 to 59 Months in Porto Alegre, Brazil. London: London School of Hygiene and Tropical Medicine/ University of London.

DRACHLER, M. L.; BOBAK, M.; RODRIGUES, L.; AERTS, D. R. G. C.; LEITE, J. C. C.; DANOVA, J. \& $K R I Z, B$, 2002. The role of socioeconomic in differences in height of pré-school children within and between the Czech Republic and Southern Brazil. Central European Journal of Public Heath, 10:135-141.

D'SOUZA, R. M., 1997. Housing and environmental factors and their effects on the health of children in the slums of Karachi, Pakistan. Journal of Biosocial Science, 29:271-281.

ENGSTROM, E. M. \& ANJOS, L. A., 1999. Déficit estatural nas crianças brasileiras: Relação com condições sócio-ambientais e estado nutricional materno. Cadernos de Saúde Pública, 15:559-567.

ESREY, A. S.; FEACHEM, R. G. \& HUGHES, J. M., 1985. Interventions for the control of diarrhoeal diseases among young children: improving water supplies and excreta disposal facilities. Bulletin of the World Health Organization, 63:757-772.

FRONGILLO, E. A.; ONIS, M. \& HANSON, K. M. P., 1997. Socioeconomic and demographic factors are associated with worldwide patterns of stunting and wasting of children. Journal of Nutrition, 127:2302-2309.

GOLDSTEIN, H., 1971. Factors influencing the height of seven year old children - Results from the $\mathrm{Na}$ tional Child Development Study. Human Biology, 43:92-111.

GOLDSTEIN, H., 1995. Multilevel Statistical Models. London: Edward Arnold.

GOPALAN, C., 1987. Retraso del crecimiento: Significado y implicaciones em la política de salud pública. In: Retraso del Crecimiento Lineal em Ios Países em Vías de Desarrollo (Nestlé, org.), pp. 3437, Thailandia: Foundation Nestlé.

GROSS, R.; SCHELL, B.; MOLINA, M. C. B.; LEÃO, M. A. C. \& STRACK, U., 1989. The impact of improvement of water supply and sanitation facilities on diarrhea and intestinal parasites: A brazilian experience with children in two low-income urban communities. Revista de Saúde Pública, 23:214220.

GREENLAND, S. \& ROTHMAN, K., 1998. Fundamentals of epidemiologic data analysis. In: Modern Epidemiology (K. Rothman \& S. Greenland, ed.), pp. 201-229, Philadelphia: Lippincott Williams \& Wilkins.

GUIM ARÃES, L. V.; LATORRE, M. R. D. O. \& BARROS, M. B. A., 1999. Fatores de risco para a ocorrência de déficit estatural em pré-escolares. Cadernos de Saúde Pública, 15:605-615.

GULDAN, G. S.; ZEITLIN, M. F.; BEISER, A. S.; SUPER, C. M.; GERSHOFF, S. N. \& DATTA, S., 1993. Maternal education and child feeding practices in rural Bangladesh. Social Science and Medicine, 36:925935.

HAAS, J. D.; MURDOCH, S.; RIVERA, J. \& MARTORELL, R., 1996. Early nutrition and later physical work capacity. Nutrition Reviews, 54:S41-S48.

HERNANDEZ-DIAZ, S.; PETERSON, K. E.; DIXIT, S.; HERNÁNDEZ, B.; PARRA, S.; BARQUERA, S.; SEPÚLVEDA, J. \& RIVERA, J. A., 1999. Association of maternal short stature with stunting in Mexican children: Common genes vs common environment. European Journal of Clinical Nutrition, 53:938-945.

IBGE (Fundação Instituto Brasileiro de Geografia e Estatística), 1983. Metodologia do Censo Demográfico de 1980. Rio de Janeiro: IBGE.

IMMINK, M. D. C. \& PAYONGAYONG, E., 1999. Risk analysis of poor health and growth failure of children in the central highlands of Guatemala. Social Science and Medicine, 48:997-1009.

ISSLER, R. M.; GIUGLIANI, E. R.; KREUTZ, G. T.; MENESES, C. F.; JUSTO, E. B.; KREUTZ, V. M. \& PIRES, M., 1996. Poverty levels and children's health status: Study of risk factors in a urban population of low socioeconomic level. Revista de Saúde Pública, 30:506-511.

JELLIFFE, D. B., 1968. Evaluación del Estado de Nutrición de la Comunidad. Série de Monografias 53. Geneva: Organización Mundial de la Salud.

KAC, G., 1999. Tendência secular em estatura: U ma revisão de literatura. Cadernos de Saúde Pública, 15:451-461.

KROMEYER, K.; HAUSPIE, R. C. \& SUSANNE, C., 1997. Socioeconomic factors and growth during childhood and early adolescence in Jena children. Annals of Human Biology, 24:343-353.

LEI, D. L. M.; CHAVES, S. P.; LERNER, B. R. \& STEFANINI, M. L. R., 1995. Retardo do crescimento físico e aproveitamento escolar em crianças do Município de Osasco, área metropolitana de São Paulo, Brasil. Cadernos de Saúde Pública, 11:238245.

MALTA, D. C.; GOULART, E. M. A. \& COSTA, M. F. L., 1998. Estado nutricional e variáveis sócio-econômicas na repetência escolar: Um estudo prospectivo em crianças da primeira série em Belo Horizonte, Brasil. Cadernos de Saúde Pública, 14: 157-164.

MARTORELL, R.; KHAN, L. K. \& SCHROEDER, D. G., 1994. Reversibility of stunting: epidemiological findings in children from developing countries. European Journal of Clinical Nutrition, 48:S45S47.

MONTEIRO, C. A., 1988. Saúde e Nutrição das Crianças de São Paulo: Diagnóstico, Contrastes Sociais eTendências. São Paulo: Editora Hucitec.

MONTEIRO, C. A. \& BENÍCIO, M. H., 1987. Estudo das condições de saúde das crianças do Municí- 
pio de São Paulo, SP (Brasil). Revista de Saúde Pública, 21:380-386.

MUÑIZ, J. A. G. \& RUBI, L. A., 1988. Escolaridad de la madre y condiciones ambientales em la morbidad, la mortalidad y el desarrollo del niño. Revista Cubana de Pediatría, 60:974-992.

NCHS (National Center for Health Statistics), 1977. Growth Curves for Children Birth-18 Years. Hyattsville: National Center for Health Statistics.

NÓBREGA, F. J.; BRASIL, A. L. D.; VÍTOLO, M. R.; LOPEZ, F. A. \& LOPEZ, L. A., 1991. Estudo da influência do peso de nascimento, de variáveis maternas e sócio-econômicas na determinação da estatura em crianças. Jornal de Pediatria, 67:111-118.

PARKER, D. R.; LAPANE, K. L.; LASATER, T. M . \& CARLETON, R. A., 1998. Short stature and cardiovascular disease among men and woman from two southeastern New England communities. International Journal of Epidemiology, 27:970-975.

PREBEG, Z., 1998. Changes in growth patterns in Zagreb school children related to socioeconomic background over the period 1973-1991. Annals of Human Biology, 25:425-439.

RICCI, J. A. \& BECKER, S., 1996. Risk factors for wasting and stunting among children in Metro Cebu, Philippines. American Journal of Clinical Nutrition, 63:966-975.

SCRIMSHAW, N. S. \& SANGIOVANNI, J. P., 1997. Synergism of nutrition, infection, and immunity: An overview. American Journal of Clinical Nutrition, 66:464S-477S.

SILVA, A. A. M.; GOMES, U. A., TONIAL, S. R. \& SILVA, R. A., 1999. Fatores de risco para hospitalização de crianças de um a quatro anos em São Luís, Maranhão, Brasil. Cadernos de Saúde Publica, 15: 749-757.
SMITH, A. M.; CHINN, S. \& RONA, R. J., 1980. Social factors and heigh gain of primary schoolchildren in England and Scotland. Annals of Human Biology, 7:115-124.

SPURR, G. B., 1987. Tamaño corporal, capacidad de realizar tabajos fisicos y productividad em el trabajo intenso: Es mejor más grande? In: Retraso del Crecimiento Lineal em Ios Países em Vías de Desarrollo (Nestlé, org.), pp. 25-29, Thailandia: Foundation Nestlé.

VICTORA, C. G.; BARROS, F. C. \& VAUGHAN, J. P., 1988. Epidemiologia da Desigualdade: Um Estudo Longitudinal de 6.000 Crianças Brasileiras. São Paulo: Editora Hucitec.

VICTORA, C. G.; HUTTLY, S. R.; BARROS, F. C.; LOM BARDI, C. \& VAUGHAN, J. P., 1992. Maternal education in relation to early and late child health outcomes: findings from a Brazilian cohort study. Social Science and Medicine, 34:899-905.

VICTORA, C. G.; VAUGHAN, J. P.; KIRKWOOD, B. R.; MARTINES, J. C.; BARCELOS, L. B., 1986. Risk factors for malnutrition in Brazilian children: The role of social and environmental variables. Bulletin of World Health Organization, 64:299-309.

WATERLOW, J. C., 1994. Causes and mechanisms of linear growth retardation (stunting). European Journal of Clinical Nutrition, 48:S1-S4.

WEINBERG, C. R., 1993. Toward a clearer definition of confounding. Americam Journal of Epidemiology, 137:1-8.

WHO (World Health Organization), 1995. Physical Status: The U se and Interpretation of Anthropometric Indicators of Nutritional Status. WHO Technical Report Series 854. Geneva: WHO.

Recebido em 29 de julho de 2002

Versão final reapresentada em 6 de maio de 2003

Aprovado em 14 de agosto de 2003 